\title{
A primary care study of dentists' and doctors' knowledge of oral cancer
}

\author{
Primary care clinicians' knowledge of oral cancer: a study of dentists and doctors in the North East of England \\ by M. Greenwood and R. J. Lowry Br Dent J 2001; 191:510-512
}

\section{Objective}

To compare the knowledge of oral cancer and related issues of general dental and general medical practitioners (GDPs and GMPs).

\section{Design}

A questionnaire was sent to 420 primary care clinicians, half to dentists and half to doctors. Responses to the questionnaire were analysed using odds ratios (OR) and $95 \%$ confidence intervals $(\mathrm{CI})$.

\section{Results}

The response rate was $68.1 \%$ for GDPs and $71.9 \%$ for GMPs. Dental practitioners were significantly more likely to have diagnosed cases of oral cancer than medical practitioners $(\mathrm{OR}=2.68,95 \% \mathrm{CI} 1.6,4.4)$. Important differences arose between the groups in terms of risk factor knowledge and clinical examination.

\section{Conclusion}

This study demonstrates that whilst the knowledge level of both groups is generally good, there are some discrepancies. These relate mainly to risk factor knowledge and clinical examination techniques.

\author{
In Brief \\ - This study compares basic knowledge of oral cancer of \\ general medical practitioners and general dental \\ practitioners working in primary care. \\ - Knowledge levels are important since either group of \\ practitioners may see such patients at first presentation. \\ - Dental practitioners were significantly more likely to name \\ alcohol as a risk factor than medical practitioners. Dental \\ practitioners showed a preference for examining areas \\ related to the tooth bearing or potentially denture bearing \\ tissues rather than some of the more high risk sites \\ eg floor of mouth. \\ - Knowledge levels were generally good but there were \\ some important differences mainly relating to risk factor \\ knowledge and clinical examination techniques.
}

\section{Comment}

7 his study uses a prospective question1 naire to explore the relative core knowledge of general dental (GDPs) and medical practitioners (GMPs) about oral cancer. Practitioners were selected randomly from FHSA lists in and around Newcastle upon Tyne and Teesside. The response rate was $68.1 \%$ for GDPs and $71.9 \%$ for GMPs, allowing a full statistical analysis.

Oral cancer is unique in presenting to two major groups of primary care practitioners. Although comparative studies of the knowledge base of these two groups have been undertaken in the USA this is a relatively unexplored area in the UK. The study uses a simple but elegantly designed questionnaire to test key areas of knowledge.

The significant findings are that GDPs were more likely to have diagnosed a case of oral cancer than GMPs. Both groups showed knowledge of habits predisposing to oral cancer. GDPs were significantly more likely to list alcohol as a risk factor, but both groups strongly identified smoking.

Differences were observed in clinical examination. GDPs showed a preference for examining areas relating to the tooth bearing, or potential denture bearing tissues. Why this should be so was unclear but might be related to their relative ease and familiarity with the tooth bearing areas. The result is that some of the more high-risk sites might not be so thoroughly examined. GDPs were more knowledgeable than GMPs about premalignant lesions and the ways in which oral cancer might present, probably because of their greater exposure to the teaching of oral pathology. The primary treatment modalities, surgery and radiotherapy were identified but chemotherapy was cited by $60.8 \%$ of GDPs and $32.5 \%$ of GMPs. Chemotherapy has a developing role but as yet it is not generally considered to be a primary treatment modality. Referral pathways were appropriate with the majority referring to specialists in oral \& maxillofacial surgery.

The study is reassuring in that it confirms that GDPs and GMPs are informed about risk factors for oral cancer and are aware of patterns of clinical presentation. One of the authors' concern relates to the way in which GDPs were noted to examine the oral cavity. Other studies have indicated that the majority of dental practitioners routinely carry out screening of the oral mucosa for premalignant and malignant lesions. ${ }^{1-3}$ For this to be effective, the oral examination needs to fully assess the areas of mucosa most at risk of malignant change. Providing the appropriate sites are fully assessed GDPs have a key role in opportunistic screening for oral cancer.

\section{Sheila E. Fisher}

Senior Lecturer/Hon. Consultant in Oral and Maxillofacial Surgery, Leeds Dental Institute, University of Leeds

1. Scully C, Malamos D, Levers B G H, Porter S R. Prime S R.Sources and patterns of referral of oral cancer: role of general dental practitioners. BrMed J 1986; 293: 599-602.

2. Cowan C G, Gregg T A, Kee F.Prevention and detection of oral cancer: the views of primary care dentists in Northern Ireland. Br Dent J 1995; 179: 338-342.

3. Field B A, Morrison T, Darling A L, Parr T A, Zakrzewska J M.Oral mucosal screening as an integral part of routine dental care. $\mathrm{Br}$ Dent $J$ 1995; 179: 262-266. 